\title{
Look to the future
}

\section{Jon Karlsson ${ }^{4} \cdot$ Roland Becker $^{3} \cdot$ Volker Musahl $^{2} \cdot$ Michael T. Hirschmann $^{1}$}

Published online: 26 November 2018

(c) European Society of Sports Traumatology, Knee Surgery, Arthroscopy (ESSKA) 2018

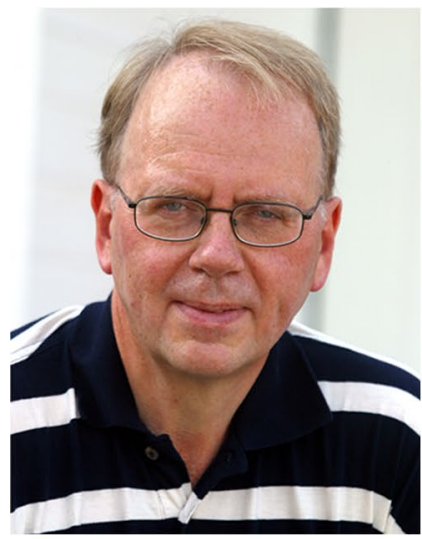

Jon Karlsson

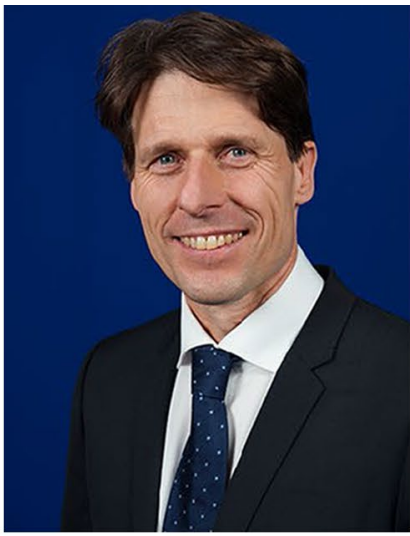

Roland Becker

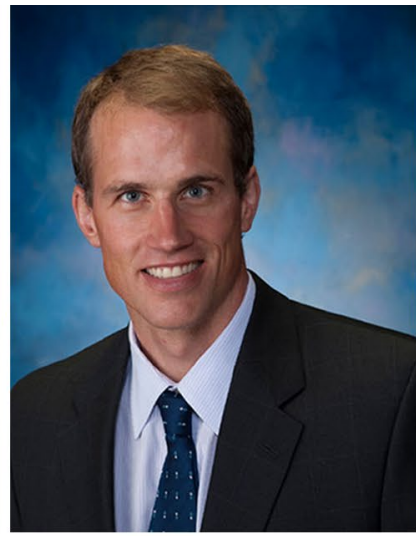

Volker Musahl

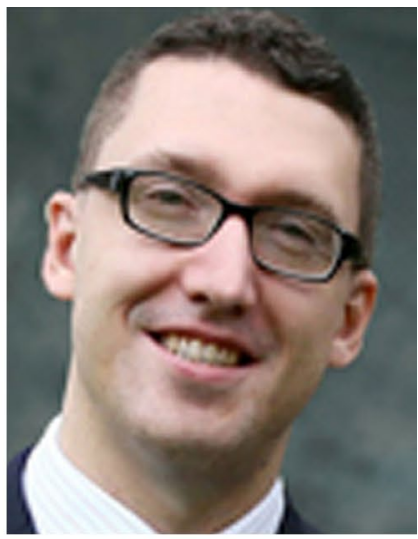

Michael T. Hirschmann

Jon Karlsson

jon.karlsson@vgregion.se

1 Department of Orthopaedic Surgery and Traumatology, Kantonsspital Baselland (Bruderholz Liestal, Laufen), 4101 Bruderholz, Switzerland

2 Department of Orthopaedic Surgery, Center for Sports Medicine, UPMC Rooney, Pittsburgh Medical Center, 3200 S Water Street, Pittsburgh, PA 15203, USA

3 Department of Orthopedics and Traumatology, Centre of Joint Replacement, Hospital Brandenburg, Medical School "Theodor Fontane", Hochstrasse 2, 14770 Brandenburg, Germany

4 Department of Orthopaedics, Sahlgrenska Academy, University of Gothenburg, Sahlgrenska University Hospital, Mölndal, Gothenburg 431 80, Sweden 


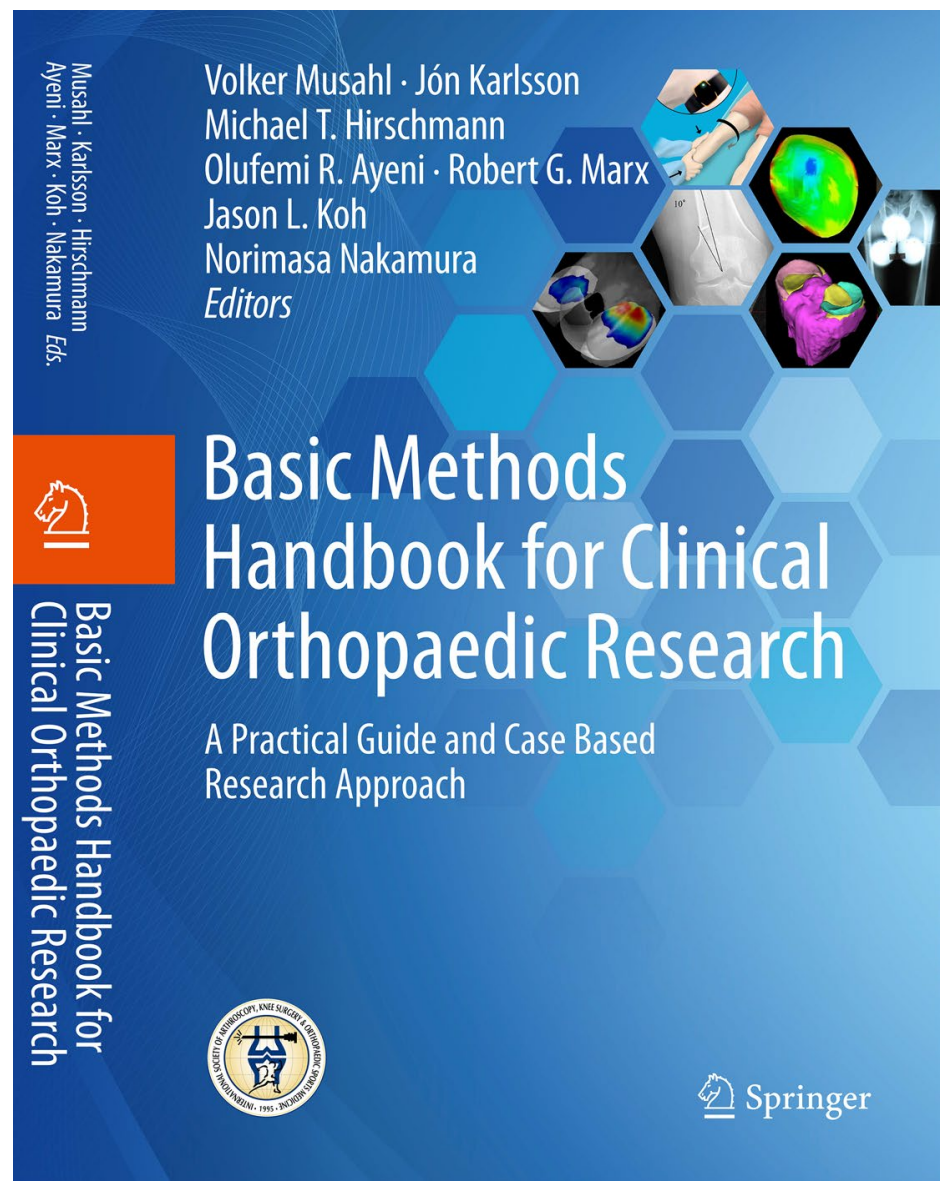

Yet, another year has passed. As usual, we would like to thank everyone who is involved in the journal for the tireless work you all do. The reviewers, who provide top-class reviews over and over again, are the backbone of the journal. We know that it takes time to review. We also know that it is unglamorous work and we are well aware that we probably ask many of our best reviewers too often. However, a good review is probably the best thing that can happen to a manuscript and many times a reviewer is also an author to a paper or papers published in the journal. In addition, by reviewing, you learn and you become a better author. We have repeatedly seen that the best reviews are in fact done by the best authors. Therefore, all great reviewers please keep up the good work and we look forward to receive your submissions as authors next year.

Overall, KSSTA has a standing of a strong scientific journal, which makes us happy as we look to a bright future. We feel that the journal is now well established as a +3 Impact Factor journal. We admit that we were somewhat disappointed that the Impact Factor stayed the same as in 2017, but this only means that we need to work even harder and look to the future. With even better papers published, better reviews and better Editorial work, we will see an improved
Impact Factor in the years to come, so we are not worried. Our ranking is high and we stay in the Top-10 range in Sports Sciences and Orthopaedics. This is wonderful news.

How can we improve? First of all, we have even further raised the bar for acceptance, and currently, we reject between 70 and $80 \%$ of all submitted manuscripts. We often do this using the "rapid reject" process, where manuscripts are rejected already at Editorial stage level, within a week or so. The "rapid reject" always happens after two of the Editors have read the manuscript. The question is why we reject already at this early stage. The reason for this is always manuscript quality. We mainly reject manuscripts with limited scientific news value, limited cohorts, lack of IRB Approvals and problems with statistics. The most common problem is related to small cohorts and subgroup analysis, leading to type-II statistical errors. Another problem is limited clinical impact. We feel that this approach is good, not only for the journal, but also for the authors, as they receive a quick response from the journal. In fact, we have received very few complaints from authors related to the "rapid reject" process.

The Journal of Experimental Orthopaedics (JEO) is continuing to get stronger. This is possible due to the constant increase in submissions. However, this is not only due to the high number of submissions, but also due to the fact 
that KSSTA now is solely a clinical journal, and therefore, the experimental work is directed to JEO. We feel that this has been successful, and we will continue to work in this direction.

This year, the number of submissions has increased to 1800 compared with 1600 during the last 2 years. The increased number of submissions increases the workload on the Editors and the Editorial Office. Therefore; starting in January 2019, there will be two people working for the journal at the Editorial Office in Luxembourg.

To increase the collaboration with the reviewers, we stage a journal reviewer course during the ESSKA congresses, last time in Glasgow this year. The course was very well attended, and was more interactive than ever before-a good sign!

What lies ahead? First of all, we will increase the collaboration with the largest sports traumatology/arthroscopy societies in Europe. Staring January 2019 with AGA (Germany) and SFA (France), we will add two Associate Editors, who will take care of this project. Therefore, we welcome Peter Angele and Nicolas Pujol in these positions, respectively. We are sure that this project will be strong and will help lead the journal forward in a positive direction.

Several scientific journals have in the last few years developed a new online publication line, for instance, American Journal of Sports Medicine (AJSM) who have been very successful in creating OJSM (Orthopaedic Journal of Sports Medicine). Other journals have followed. Due to the increasing number of submissions, KSSTA will move in the same direction. We simply need to find place to publish all good manuscripts submitted to the journal. At the same time, we feel that 330 pages/month is the maximum size of the journal, and we have no plans to increase the number of pages. We are currently in negotiations with Springer, our publisher about the most relevant business model, online or paper or hybrid. The plan is to launch the new journal during the ESSKA Speciality Days in Madrid in November 2019.

This group of Editors has worked for the journal for years now and both Michael Hirschmann and Volker Musahl are now Deputy Editors along with Roland Becker, who will continue to support the journal in his new position as second vice president of ESSKA. In this way, the excellent communication between the ESSKA Board and the Editors will be even stronger.

Our goal is always good research, and as Editors, we strive to improve the scientific value of papers published in the journal. The review process is-due to this reasonoften long and cumbersome. Almost all manuscripts that are eventually published undergo at least two revisions and some even three or four. As already mentioned, a good review makes the manuscript a better paper, and therefore, authors should always do their best to comply with the review process. In fact, almost all second, third, and fourth revisions are done by the Editor-in-Chief. Our goal is to publish the best papers possible. However, we also do more, as during the last year, we have worked hard on a new "Basic Methods Handbook for Clinical Orthopaedic Research", aimed at clinicians. This book will be published as a joint effort by ESSKA and ISAKOS at the beginning of 2019. It will be over 600 pages and will cover every aspect of clinical research. We worked tirelessly to make this book an easy read with numerous clinical case examples, fact boxes, and take home messages for every chapter. This book will help clinical scientists to improve their scientific knowledge, perform better studies, and write better papers. We are proud of this work and we are excited to bring this work to you, our readers, in 2019.

Finally, we, the Editors of KSSTA, would like to thank readers, authors, and reviewers for the year that is almost passed. We wish you a peaceful holiday season, may all of you enjoy time with your families and recharge for a productive, enjoyable, and scientifically rewarding year 2019.

\section{Acknowledgement to referees}

Henrik Aagaard
Pierre Abadie
Jeffrey Abrams
Andrea Achtnich
Paul Ackermann
Nobuo Adachi
Paolo Adravanti
Jens Agneskirchner
Rintje Agricola
Mattias Ahldén
Sufian Ahmad
Philipp Ahrens
Eduard Alentorn-Geli
Bogdan Ambrozic
Jack Andrish
Pierluigi Antinolfi
Daisuke Araki
Paulo Araujo
Elizabeth Arendt
Paolo Arrigoni
Rene Attal
Jesper Augustsson
Corrado Bait
Peter Balcarek
Maurice Balke
Adad Baranto
Björn Barenius
Kristoffer Barfod
Johannes Barth
John Bartlett


Cecile Batailler

Thomas Bauer

Mike Baums

Philippe Beaufils

Christoph Becher

Johannes Beckmann

Henrik Behrend

Knut Beitzel

Eva Bengtsson Mostrom

Massimo Berruto

Onur Bilge

Erkal Bilgic

Kerem Bilsel

Rudi Bitsch

Hanna Bjørnsson Hallgren

Lars Blønd

Gerrit Bode

Tommaso Bonanzinga

Bert Boonen

Ulrich Bosch

Kristín Briem

Martijn Brinkman

Matthias Brockmeyer

Robert Brophy

Annelie Brorsson

Reinoud W. Brouwer

Nicolaas Budhiparama

Paolo Bulgheroni

Jeremy Burnham

Bruce Burnham

Carlo Camathias

Stefano Campi

Mike Carmont

Antonio Cartucho

Enric Castellet

Etienne Cavaignac

Chong Bum Chang

Ofir Chechik

Antonia Chen

Cheng-Kung Cheng

Vasileios Chouliaras

Martin Clauss

Riccardo Compagnoni

Vincenzo Condello

Fabio Conteduca

Ryan Coughlin

P. Maxwell Courtney

James Cowan

Davide Cucchi

Jari Dahmen

Kiriakos Daniilidis

Thomas De Bo

Ricardo de Casas
Marco De Gori

Darren de SA

Lieven De Wilde

Kamal Deep

Koen Defoort

Masataka Deie

Francesco Della Villa

Brian Devitt

Paolo di Benedetto

Tobias Dietrich

Simon Donell

Mahmut Doral

Klaus Draenert

Björn Drews

Matej Drobnic

Jon Drogset

Lutz Duerselen

Felix Dyrna

Turgay Efe

Lars Ejerhed

Nathan Endres

Nathan Endres

Bengt Eriksson

Karl Eriksson

Lucio Ernlund

Aidin Eslam Pour

João Espregueira-Mendes

Max Ettinger

Simon A. Euler

Denise Eygendaal

Anna Falkowski

Filippo Familiari

Jack Farr

Peter Fauno

Mario Ferretti

Matthias Feucht

Christian Fialka

Giuseppe Filardo

Christian Fink

Radu Fleaca

Francisco Forriol

Francesco Franceschi

Karl-Heinz Frosch

John Fulkerson

Pablo Gelber

Christian Gerhardt

Wayne Gersoff

Alan Getgood

Thomas Gill

Johannes Glasbrenner

Mark Glazebrook

John Gliatis

Alberto Gobbi 
Alli Gokeler

Pedro Gomez

Enrique Gomez-Barrena

Andreas Gomoll

Vincent Gouttebarge

Alberto Grassi

Karin Grävare Silbernagel

Elliot Greenberg

Stefan Grote

Daniel Guenther

Clemens Gwinner

Hanna Gyllensten

Carl Haasper

William Hage

Martin Hägglund

Tamas Halasi

Andreas Halder

Eric Hamrin Senorski

Michael Hantes

Naoki Haraguchi

Horia Haragus

Arsi Harilainen

Joerg Harrer

Elmar Herbst

Philipp Heuberer

Stefan Hinterwimmer

Anna Hirschmann

Alfred Hochrein

Siegfried Hofmann

MaCalus Hogan

Yuichi Hoshino

Stephen Howell

Christophe Hulet

Ingrid Hultenheim Klintberg

Mark Hutchinson

Andreas Imhoff

Eivind Inderhaug

Takanori Iriuchishima

Andreas Ivarsson

Kaywan Izadpanah

Per-Mats Janarv

Rob Janssen

Timo Jarvela

Jayadeep Jayachandran

Mislav Jelic

Jean-Yves Jenny

Jae-Heon Jeong

Joerg Jerosch

Urban Johnson

Peter Kälebo

Mustafa Karahan

Jan Christoph Katthagen

Jefferey Kay
Defne Kaya

Mahmut Enes Kayaalp

John Kennedy

Willem A. Kernkamp

Oliver Kessler

Moin Khan

Shwan Khoschnau

Christoph Kittl

Gunnar Knutsen

Koichi Kobayashi

Baris Kocaoglu

Mininder Kocher

Izaäk Kodde

Hideyuki Koga

Jason Koh

Ioannis Kostogiannis

Rover Krips

Michael Krogsgaard

Werner Krutsch

Rik Kundra

Robert LaPrade

Ricardo Larrainzar-Garijo

Lior Laver

Keith Lawhorn

Sang Hak Lee

Peter Leeuw

Pisit Lertwanich

Ofer Levy

Guoan Li

Sven Lichtenberg

Michael Liebensteiner

Dennis Liem

Albert Lin

Martin Lind

Anders Lindahl

Daphne Ling

Sverre Löken

Nicola Lopomo

Olaf Lorbach

Joerg Luetzner

Bent Lund

Mari Lundberg

Stephen Lyman

Jack Lysholm

Jeffrey Macalena

Henning Madry

Nicola Maffulli

Robert Magnussen

Mahir Mahirogullari

Konstantinos Makridis

Giulio Maria Marcheggiani Muccioli

Fabrizio Margheritini

Antonio Marmotti 
Frank Martetschläger

Vladimir Martinek

Inigo Martinez

Philippe Massin

Dominic Mathis

Craig Mauro

Oliver May

Hermann-Otto Mayr

Khaled Meknas

Jacques Menetrey

Gilbert Moatshe

Stefan Mogos

Håvard Moksnes

Juan Monllau

Lukas Moser

Caroline Mouton

Andreas Mueller

Thomas Muellner

Lars Muller

Sebastian Müller

Raman Mundi

Takeshi Muneta

Peter Myers

Truls Straume Næsheim

Kanto Nagai

Takumi Nakagawa

Atsuo Nakamae

Norimasa Nakamura

Richard Napier

Wolfgang Nebelung

Manfred Nelitz

Katarina Nilsson-Helander

John Nyland

Shawn O'Driscoll

Matthieu Ollivier

Nicklas Olsson

Patrick Orth

Christian Owesen

Geert Pagenstert

Nirav Pandya

Pericles Papadopoulos

Sebastien Parratte

Rahul Patel

Thilo Patzer

Thierry Pauyo

Luigi Pederzini

Yun Peng

Hélder Pereira

Andreas Persson

Devin Peterson

Maximilian Petri

Frank Petrigliano

Frederic Picard
Martin Pietsch

Tom Piscaer

Kevin Plancher

Chadwick Prodromos

Nicolas Pujol

Acacio Ramos

Filippo Randelli

Pietro Randelli

Per Henrik Randsborg

Bjoern Rath

Valentin Rausch

Mikel Reilingh

Tommaso Roberti di Sarsina

Scott Rodeo

Justin Roe

Philip Roessler

Dieter Rosenbaum

Claudio Rosso

Benjamin Rothrauff

Jan Harald Røtterud

Miguel Ruiz Iban

Octav Russu

Sofia Ryman Augustsson

Patrick Sadoghi

Mo Saffarini

Adnan Saithna

Björn Salomonsson

Gonzalo Samitier

Kristian Samuelsson

Mikael Sansone

Alfredo Schiavone Panni

Oliver Schindler

Phillip Schottle

Steffen Schröter

Elvire Servien

Andrew Sheean

Seth Sherman

Konsei Shino

Cecilia Signorelli

Petri Sillanpaa

Luminita Simion

Einar Andreas Sivertsen

Inge Skråmm

Daniel Smolen

Francesc Soler

Eirik Solheim

Bertrand Sonnery-Cottet

Anne Kathrine Sørensen

Gunter Spahn

Tim Spalding

Pietro Spennacchio

Anders Stålman

Christian Stärke 
Robert Steensen

Nicholas Stergiou

Sjoerd Stufkens

Eleonor Svantesson

Masato Takao

Simon Thompson

Fritz Thorey

Jonas Thorlund

Thomas Tischer

Jaap Tolk

Marc Tompkins

Philippe Tscholl

Soshi Uchida

Ewould Van Arkel

Christiaan van Bergen

Michel P. J. van den Bekerom

Olivier van der Meijden

Derek van Deurzen

Pim van Dijk

Carola van Eck

Nicolien Van Giffen

Jakob Van Oldenrijk

Tom van Raaij

Remco van Wensen

Michele Vasso

Alberto Ventura
Bruno Violante

Mihai Vioreanu

Andreas Voss

Bernhard Waibl

Markus Waldén

William Walsh

Patrick Weber

Kilian Wegmann

Suzanne Werner

Johannes Wiegerinck

Jaap Willems

Andy Williams

Philippe Wilmes

Diederick Wouters

Kazunori Yasuda

Shinichi Yoshiya

Alastair Younger

Lukas Zak

Frantzeska Zampeli

Alexander Zeh

Eva Zeisig

Jennifer Zellers

Junjun Zhu

Matthias Zumstein

Oskar Zupanc 